\title{
High prevalence of secondary bladder cancer in men on radiotherapy for prostate cancer: evidence from a meta-analysis
}

This article was published in the following Dove Medical Press journal: Cancer Management and Research

\author{
Shankun Zhao',* \\ Qiang $\mathrm{Xie}^{2, *}$ \\ Redian Yang ${ }^{1, *}$ \\ Jiamin Wang' \\ Chaofeng Zhang' \\ Lianmin Luo' \\ Zhiguo Zhu' \\ Yangzhou Liu' \\ Ermao Li' \\ Zhigang Zhao'
}

'Department of Urology and Andrology, Minimally Invasive Surgery

Center, Guangdong Provincial Key Laboratory of Urology, The First

Affiliated Hospital of Guangzhou

Medical University, Guangzhou,

Guangdong, China; ${ }^{2}$ Department of

Reproduction, Southern Medical

University Affiliate Dongguan People's

Hospital, Dongguan, China

*These authors contributed equally to this work
Correspondence: Zhigang Zhao

Department of Urology and Andrology, Minimally Invasive Surgery Center, Guangdong Provincial Key Laboratory of Urology, The First Affiliated Hospital of Guangzhou Medical University, Kangda Road I\#, Haizhu District, Guangzhou,

Guangdong 510230, China

Tel/fax+862034295590

Email zgzhaodr@I26.com
Objective: To assess whether radiotherapy $(\mathrm{RT})$ for prostate cancer $(\mathrm{PCa})$ was a risk factor for secondary bladder cancer (BLCa) through a meta-analysis.

Materials and methods: The MEDLINE, Embase, and the Cochrane Library were systematically searched for all studies investigating the risk of BLCa in patients with RT. The association between RT and risk of BLCa was summarized using hazard ratio with a 95\%CI. The protocol for this meta-analysis is available from PROSPERO (CRD42018090075).

Results: Overall, 619,479 participants (age: 57-79 years) were included from 15 studies, 206,852 of whom were patients who received RT. Synthesis of results indicated that RT was significantly associated with an increased risk of BLCa compared with the risk in those who received radical prostatectomy or non-RT (overall $\mathrm{HR}=1.6,95 \% \mathrm{CI}$ : $1.33-1.92, P<0.001$ ). The results were consistent when restricted to a 5-year lag period ( $\mathrm{HR}=1.84,95 \% \mathrm{CI}$ : 1.26-2.69, $P=0.002)$ and multivariable adjustment (HR=1.96, 95\%CI: $1.47-2.62, P<0.001)$, but not for 10 -year lag period $(\mathrm{HR}=1.93,95 \% \mathrm{CI}$ : $0.9-4.16, P=0.093)$ and brachytherapy subgroup (HR=1.33, 95\%CI: 0.87-2.05, $P=0.188$ ). The GRADE-profiler revealed that the rate of events of BLCa on average in the RT-patients and the non-RT control was 2,462/183,669 (1.3\%) and $4,263 / 382,761(1.1 \%)$, respectively; the overall quality of the evidence was low.

Conclusion: Patients who received RT for PCa was associated with higher risks of developing secondary BLCa compared to those unexposed to RT, but the absolute effect was low.

Keywords: prostate cancer, radiation, radiotherapy, secondary bladder cancer, radical prostatectomy

\section{Introduction}

Radical prostatectomy (RP) and radiotherapy (RT) are the two primary nonconservative therapeutic options for men diagnosed with clinically localized prostate cancer (PCa). As the two most common forms of RT, external beam radiotherapy (EBRT) and brachytherapy (BT) are chosen by $11.6 \%$ and $13.3 \%$ of PCa patients in the US, respectively. ${ }^{1}$

Through survival benefit, however, RT has been associated with various adverse effects including pelvic pain, lower urinary tract symptoms (ie, dysuria, frequency, urgency, incontinence, and hematuria), and rectal symptoms (ie, tenesmus, mucus, hematochezia, fistula, ulceration, and necrosis). ${ }^{2}$

As the adjacent organs of the prostate, ie, bladder and rectum, are vulnerable to ionizing radiation inescapably. Accumulated data from clinical studies have suggested that a specific complication for PCa patients undergoing RT is the probability of 
elevated risk of a second primary bladder cancer (BLCa). ${ }^{3,4}$ It has long been accepted that this potential association between RT and secondary BLCa might attribute to a prostate metastatic lesion invading the bladder by the radiation scatter and radiation-induced genetic alterations. ${ }^{5,6}$ However, some investigators did not support this association; they failed to find an increased risk of secondary BLCa after RT. ${ }^{7,8}$

Currently, a number of more recent publications have continually struggled to address this relationship. Nevertheless, the evidence for this potential connection is still controversial. We have therefore attempted to investigate whether RT was a risk factor for secondary BLCa. We elected to use a metaanalysis of all the available data to better answer this question.

\section{Materials and methods}

This meta-analysis was conducted in accordance with the preferred reporting items for systematic reviews and metaanalyses (PRISMA) guidelines. PRISMA checklist was shown in Table S1. The protocol for this meta-analysis was available from PROSPERO (CRD42018090075; http://www. crd.york.ac.uk/PROSPERO).

\section{Search strategy}

MEDLINE (PubMed), Cochrane Library, and the Embase were systematically searched from the inception of the databases to February 12, 2018. The search was restricted to English language and human participants. Subject headings and the text keywords were used for the search. The search strategy was illustrated in the Supplementary materials section. Data extraction was performed by two authors independently. Disagreements were resolved by consensus with a third author.

\section{Study selection}

We included all of the available epidemiologic evidence that reported the patients who received RT developing secondary BLCa compared with those patients who received RP treatment, or those not treated with RT. On the basis of patient, intervention, comparison, outcome, and study design (PICOS), the question that guided this meta-analysis was: does RT for PCa increase the risk of secondary BLCa? The PICOS evidence base used consisted of the following combinations: clinically localized $\mathrm{PCa}$ patients $(\mathrm{P})$; a history of RT for PCa, all types of RT were included (ie, EBRT, BT) (I); compared with the PCa patients with RP treatment or no EBRT (C); the diagnosis of secondary BLCa (O). We accepted all study designs (S). In addition, studies on the pertinent subjects, which provided HR estimates with $95 \%$ CIs or adequate data to allow calculation of these effect measures. The exclusion criteria included the following: (1) studies without examining the research question or that do not meet inclusion criteria; (2) without the control groups data or inappropriate grouping (RP combining RT was excluded); (3) review articles, meeting abstracts, comments, editorials, letters, case reports, or congress reports; (4) insufficient outcome data; (5) duplicated or updated data; (6) nonclinical studies, (7) studies with $<10$ participants in either the exposure or the control group. For multiple publications of the same clinical trial, only the most recent or representative study was included.

\section{Data extraction}

Two authors (SKZ and JMW) independently extracted the following data: the first authors' names, year of the publication, study regions, data source (study interval), study design, follow-up, age, sample sizes, HR, and survival information. Previous studies defined the radiation-induced secondary cancer occurring after 5 years of radiation exposure, thus we also recorded the length of the lag period from the time of treatment to the confirmation of secondary BLCa among the included studies.

\section{Quality assessment and the overall quality of the evidence}

The methodological quality assessment of the cohort study was based on the Newcastle-Ottawa Scale (NOS). In this meta-analysis, article quality of cohort study was assessed as follows: low quality $=0-3$, moderate quality $=4-6$, high quality =7-9. The GRADE approach was applied to generate absolute estimates of the risk of BLCa in patients who received RT for $\mathrm{PCa}$, evaluate and rank the overall quality of the evidence.

\section{Statistical analyses}

The strength of association between EBRT for PCa and the risk of secondary BLCa in the included studies was estimated using hazard ratio and its $95 \%$ CI. Results with a two-tail $P$-values $<0.05$ were considered statistically significant. The heterogeneity of included studies was measured by using the Cochrane $Q$ statistic and the $I^{2}$ statistic $\left(I^{2}>50 \%\right.$ was regarded to be consistent with substantial heterogeneity; $P$-value of $Q$ test $<0.10$ was considered statistically significant). Owing to the high likelihood of between-study variance for differences in study design as well as the study population, a random effects model rather than a fixed effects model was applied in the current study. Subgroup analyses were performed to further detect the origin of heterogeneity. We also conducted the sensitivity analyses by dropping one included study in 
each analysis to evaluate the individual impact on the overall pooled result. Publication bias analysis was evaluated with Begg's and Egger's tests. The statistical analysis in the present study was performed using the Stata (version 13.0, Stata Corp LP, College Station, TX, USA).

\section{Results}

\section{Literature search}

A total of 1,507 citations were identified in the initial search. Among these, 1,443 were excluded after removing duplicates, articles without examining research question, nonclinical studies, reviews, comments, and case reports. Sixty-four potentially relevant studies were retrieved for full-text review. Among them, nine studies were excluded for not including the control group; 14 for failing to meet the inclusion criteria; six for inappropriate grouping; 13 for insufficient outcome data; and seven for being previous versions of included studies. The selection process and list of full-text excluded articles are shown in Figure 1 and the Supplementary materials, respectively. In all, 15 studies $^{9-23}$ (one article reported EBRT vs RP and BT vs RP, thus being set as two studies for calculation) met the inclusion criteria and were included in the current meta-analysis. Of note, we identified seven articles ${ }^{12,24-29}$ derived from the US surveillance, epidemiology, and end results (SEER) cohort and two articles ${ }^{8,21}$ derived from the Japan Kyushu University Hospital in our literature search. These cohort studies overlapped the study population and outcomes, thus we only included the later or representative study ${ }^{12,21}$ to prevent the duplication of patients and study intervals. The information of the remaindered cohort studies from SEER and Kyushu University Hospital were shown in the bottom of Table 1.

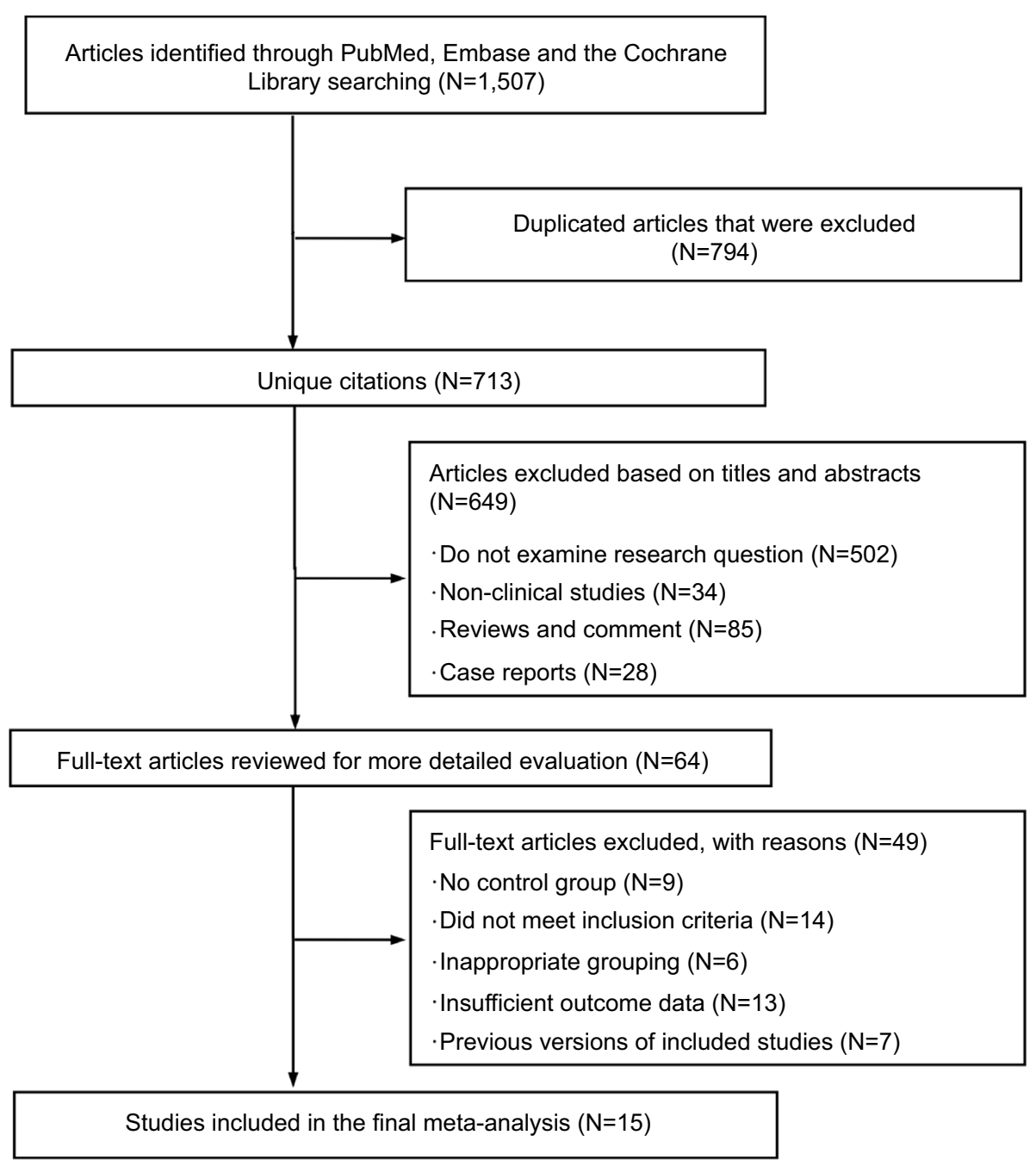

Figure I Flow chart of study selection. 


\begin{tabular}{|c|c|c|c|c|c|c|c|c|c|c|}
\hline 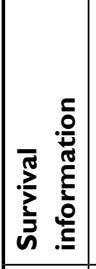 & 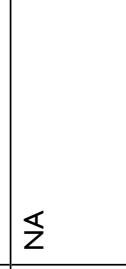 & $\frac{\mathbb{z}}{2}$ & 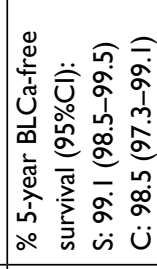 & 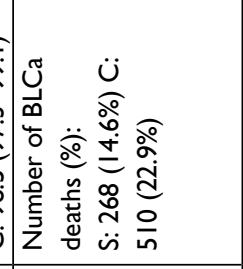 & 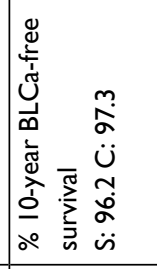 & 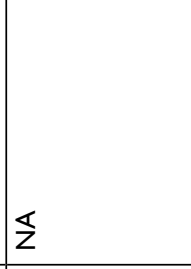 & $\frac{\$}{z}$ & $\S$ & $\underline{z}$ & 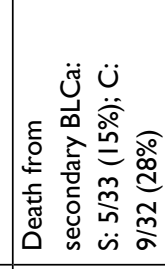 \\
\hline 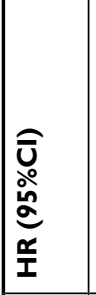 & 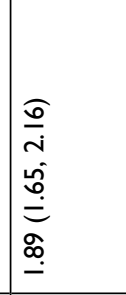 & 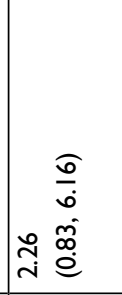 & 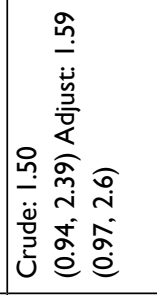 & 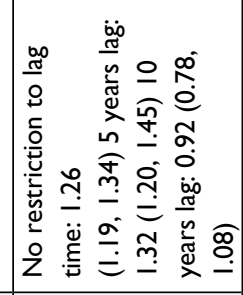 & 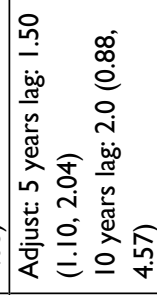 & 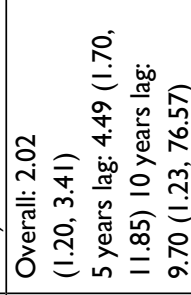 & 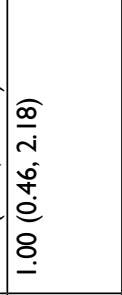 & 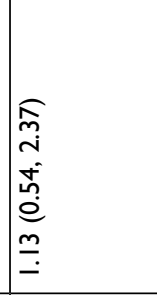 & 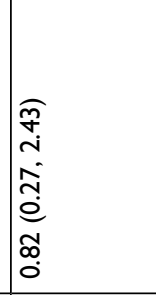 & 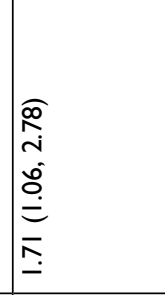 \\
\hline 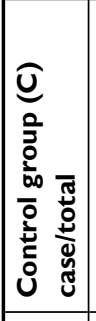 & 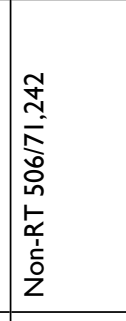 & 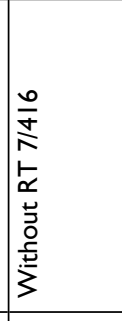 & 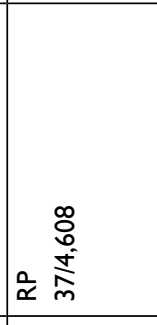 & 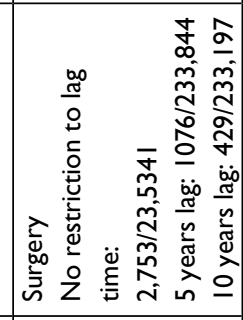 & 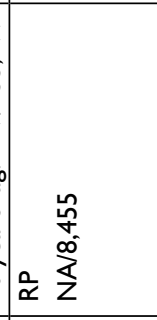 & 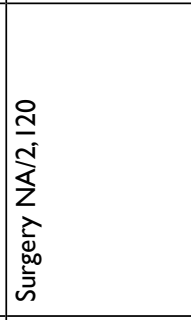 & $\mid \begin{array}{l}\bar{Q} \\
\frac{\hat{\alpha}}{0} \\
\bar{\alpha} \\
\underline{\alpha}\end{array}$ & 旁 & 递 & 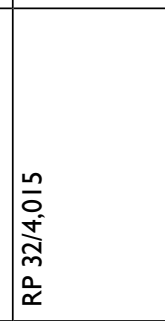 \\
\hline 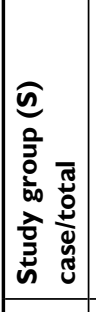 & 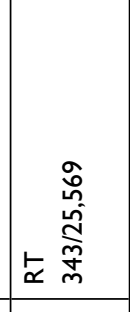 & $5 \underset{\infty}{\frac{0}{\sigma}}$ & 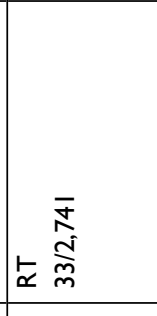 & 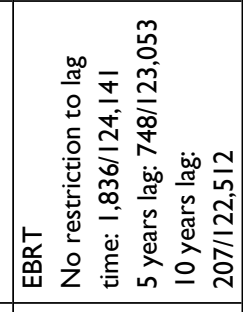 & 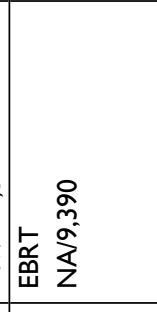 & 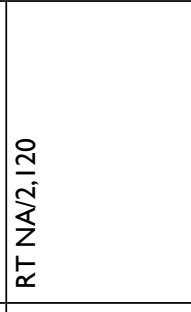 & 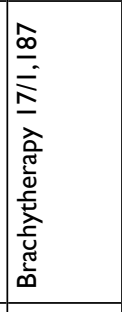 & 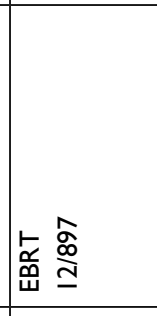 & 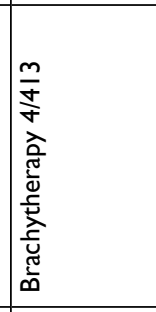 & 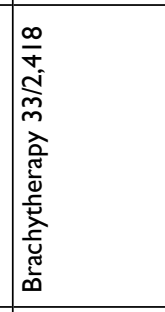 \\
\hline 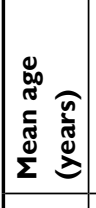 & $\begin{array}{l}\stackrel{n}{\grave{R}} \\
\stackrel{\overline{\bar{\alpha}}}{4}\end{array}$ & $\begin{array}{l}\tilde{a} \\
\infty \\
\stackrel{\bar{\alpha}}{\bar{\alpha}}\end{array}$ & $\begin{array}{l}R \\
\ddot{\overline{\bar{\alpha}}} \\
\end{array}$ & 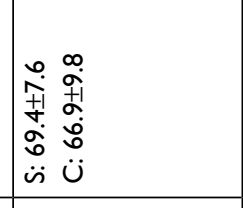 & 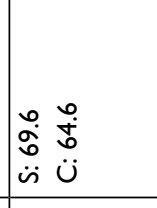 & $\mid \begin{array}{ll}\pi & R \\
\ddot{i} & u\end{array}$ & $\mid$ & 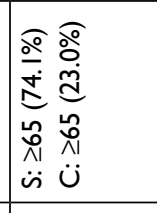 & 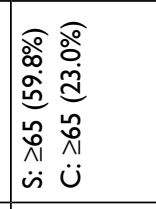 & 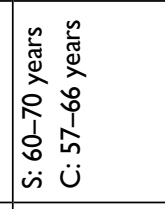 \\
\hline 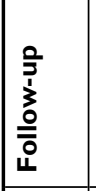 & 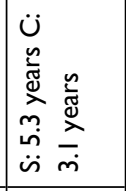 & $\frac{\mathbb{s}}{z}$ & 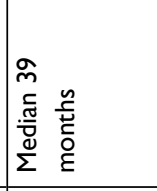 & 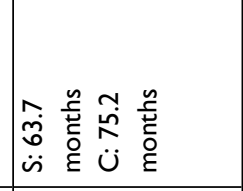 & 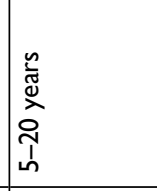 & 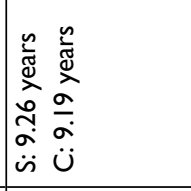 & 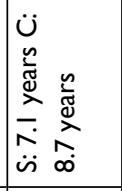 & 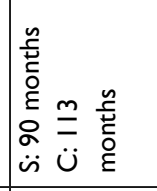 & 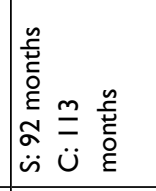 & 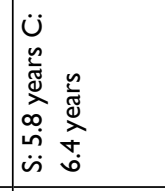 \\
\hline 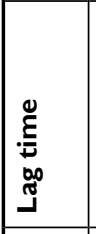 & $\frac{s}{z}$ & 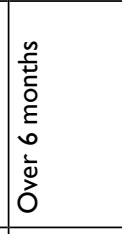 & $\underline{z}$ & 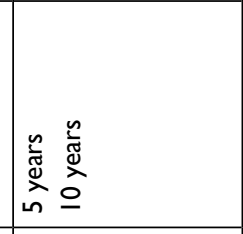 & 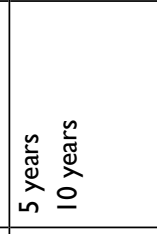 & 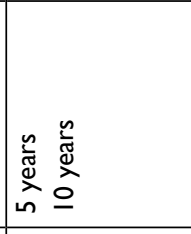 & $\underline{z}$ & 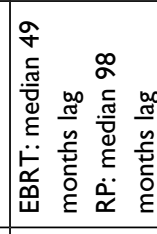 & 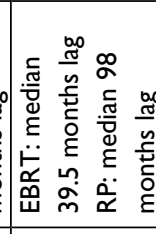 & 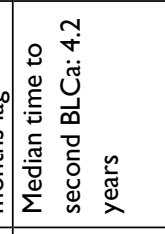 \\
\hline 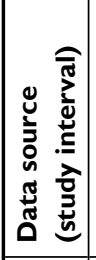 & 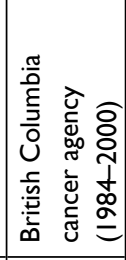 & 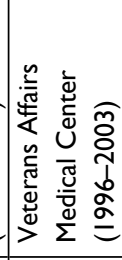 & 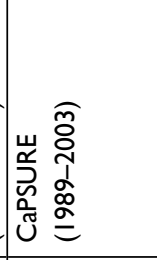 & 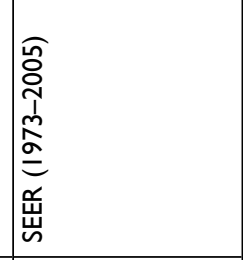 & 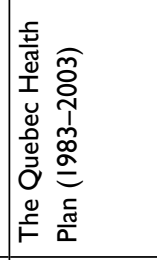 & 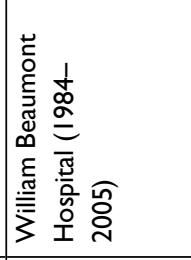 & 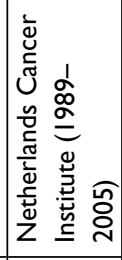 & 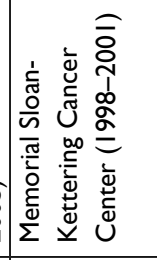 & 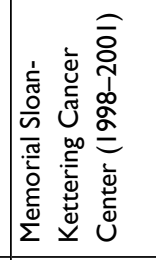 & 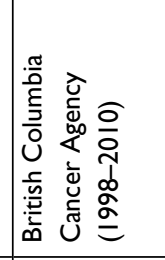 \\
\hline 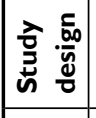 & $\begin{array}{l}\frac{4}{0} \\
\frac{0}{0} \\
0 \\
\end{array}$ & $\begin{array}{l}\frac{5}{0} \\
\text { o. } \\
\end{array}$ & \begin{tabular}{|l} 
\\
o. \\
Oे \\
\end{tabular} & $\begin{array}{l}\frac{4}{0} \\
\frac{0}{0} \\
\end{array}$ & $\begin{array}{l}5 \\
\frac{0}{0} \\
\\
\end{array}$ & 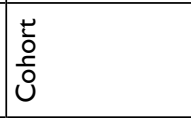 & 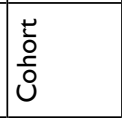 & $\begin{array}{l}\frac{5}{0} \\
\frac{0}{0} \\
\end{array}$ & $\begin{array}{l}5 \\
\frac{5}{0} \\
\\
\end{array}$ & \begin{tabular}{|l}
$\frac{t}{0}$ \\
$\frac{0}{6}$
\end{tabular} \\
\hline \begin{tabular}{|l} 
㐔 \\
जि \\
\end{tabular} & 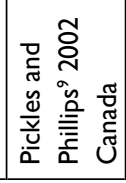 & 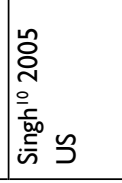 & 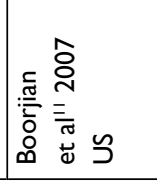 & 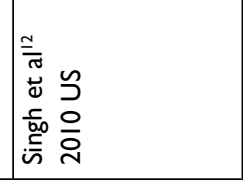 & 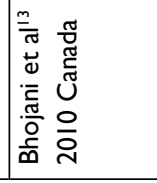 & 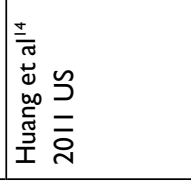 & 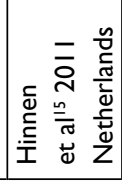 & 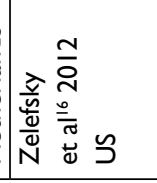 & 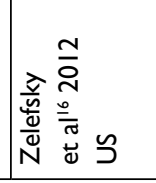 & 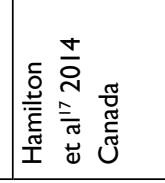 \\
\hline
\end{tabular}




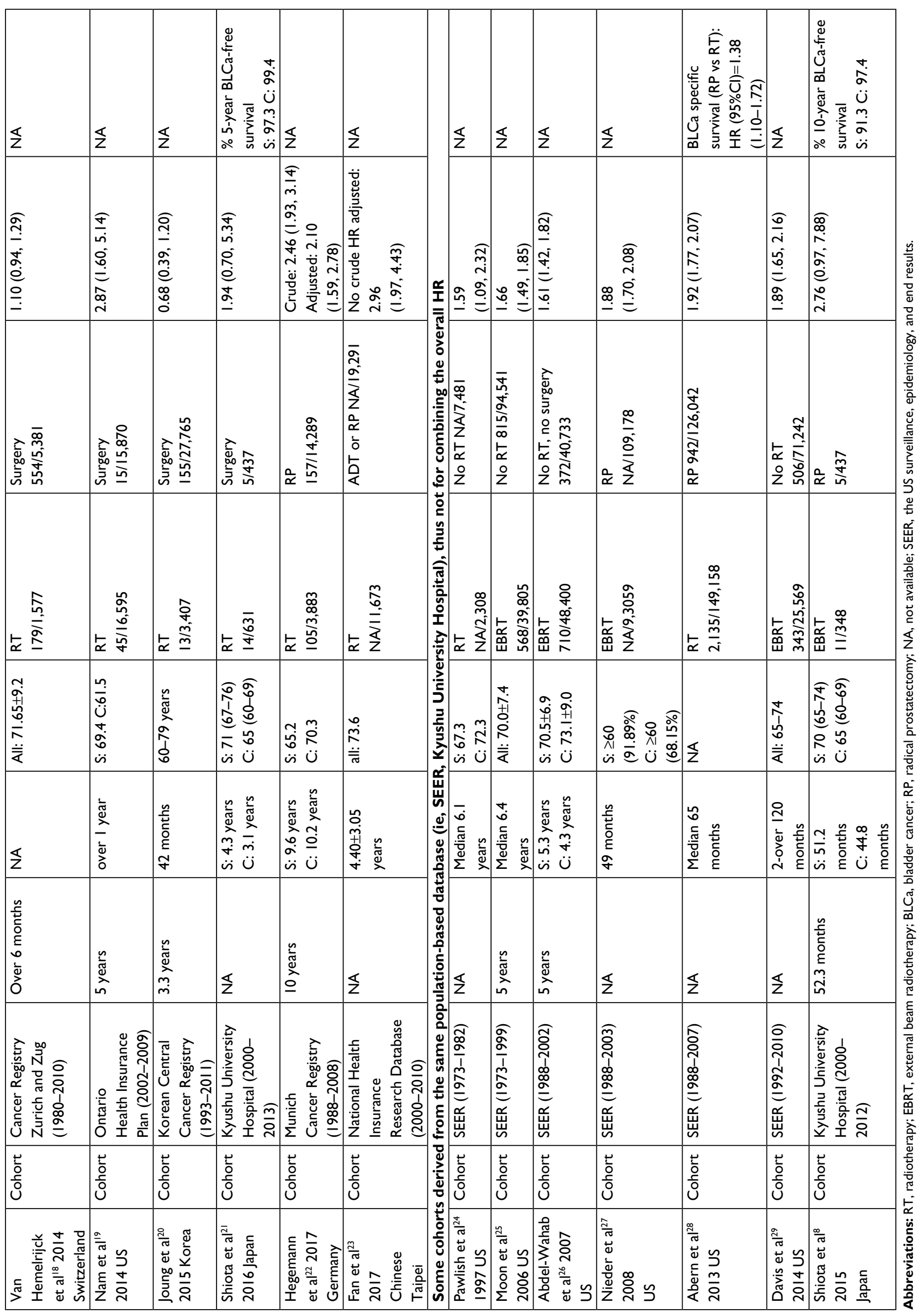




\section{Study characteristic}

The 15 included studies, published between 2002 and 2017, were all cohort designed. Of the 15 studies, nine were conducted in America, ${ }^{9-14,16,17,19}$ three in Europe, ${ }^{15,18,22}$ and three in Asia. ${ }^{20,21,23}$ Among all the included studies, a total of 619,479 individuals (mean age 60-73.6 years) were enrolled, 206,852 of whom were PCa patients who received RT, while the remaining 412,627 subjects were PCa patients who received RP treatment or non-RT. Of these included studies, two compared RT vs non-RT, eight compared RT vs RP, three compared EBRT vs RP, and three compared BT vs RP. The lag period varied between the included studies, ranging from 6 months to 10 years. The median length of follow-up ranged from 6 months to 20 years. Four studies reported the adjusted HR of the cohort study. The detail data of the included studies were summarized in Table 1.

\section{Study quality and the overall quality of the evidence}

Using the NOS, 12 studies $^{11-20,22,23}$ were considered high quality and three studies ${ }^{9,10,21}$ were judged medium quality (Table S2). The GRADE-relevant outcomes demonstrated that the rate of events of secondary BLCa on average in patients who received RT for PCa was 2,462/183,669 (1.3\%), whereas in subjects who underwent RP or without RT was 4,263/382,761 (1.1\%); the absolute effect of RT on secondary BLCa was 7 more per 1,000 (from 4 more to 10 more); the overall quality of the evidence was judged as low (Table 2).

\section{Synthesis of results}

On unadjusted analysis of the studies with no restriction to lag period, synthesis of results showed that patients who received RT for PCa were significantly associated with an increased risk of $\mathrm{BLCa}$ (16 studies; $\mathrm{HR}=1.6,95 \% \mathrm{CI}$ : 1.33 1.92, $P<0.001$; heterogeneity: $P^{2}=83.6 \%, P<0.01$ ) (Figure 2 ). The outcomes were similar when restricted to a 5 -year lag period (four studies; $\mathrm{HR}=1.84,95 \% \mathrm{CI}$ : $1.26-2.69, P=0.002$; heterogeneity: $P^{2}=76.6 \%, P=0.005$ ) (Figure $\mathrm{S} 1$ ), while no positive association between RT and secondary BLCa was found when restricted to a 10-year lag period (four studies; $\mathrm{HR}=1.93,95 \% \mathrm{CI}$ : $0.9-4.16, P=0.093$; heterogeneity: $P=93.8 \%, P<0.001$ ) (Figure $\mathrm{S} 2$ ). After multivariable adjustment, the combined results revealed that $\mathrm{PCa}$ patients who received RT had a significantly higher risk of BLCa than the non-RT subjects $(\mathrm{HR}=1.96,95 \% \mathrm{CI}: 1.47-2.62, P<0.001$; heterogeneity: $P^{2}=61.4 \%, P=0.051$ ) ( Figure $\mathrm{S} 3$ ).

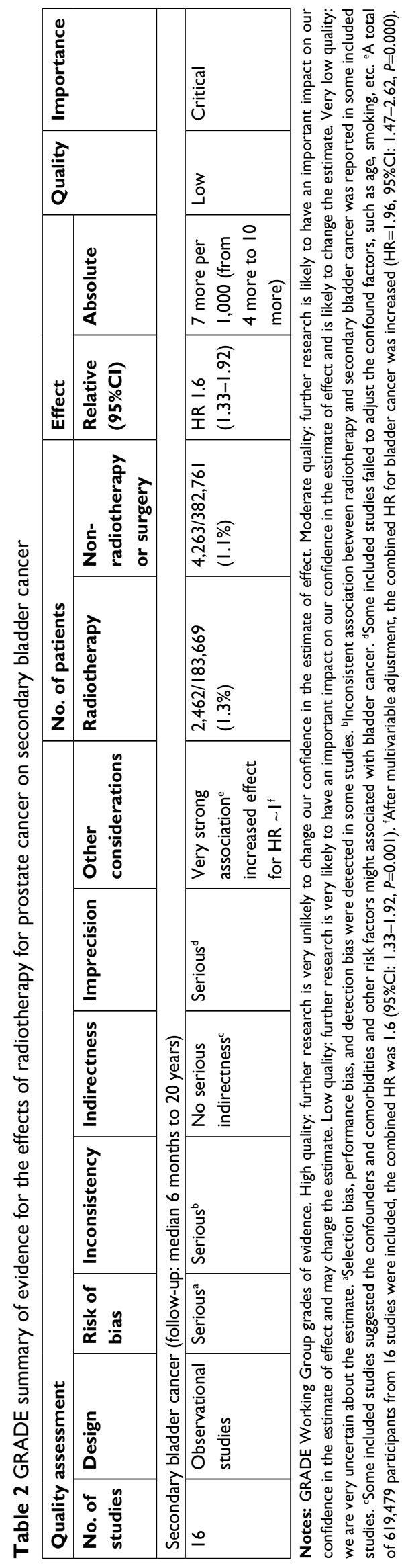




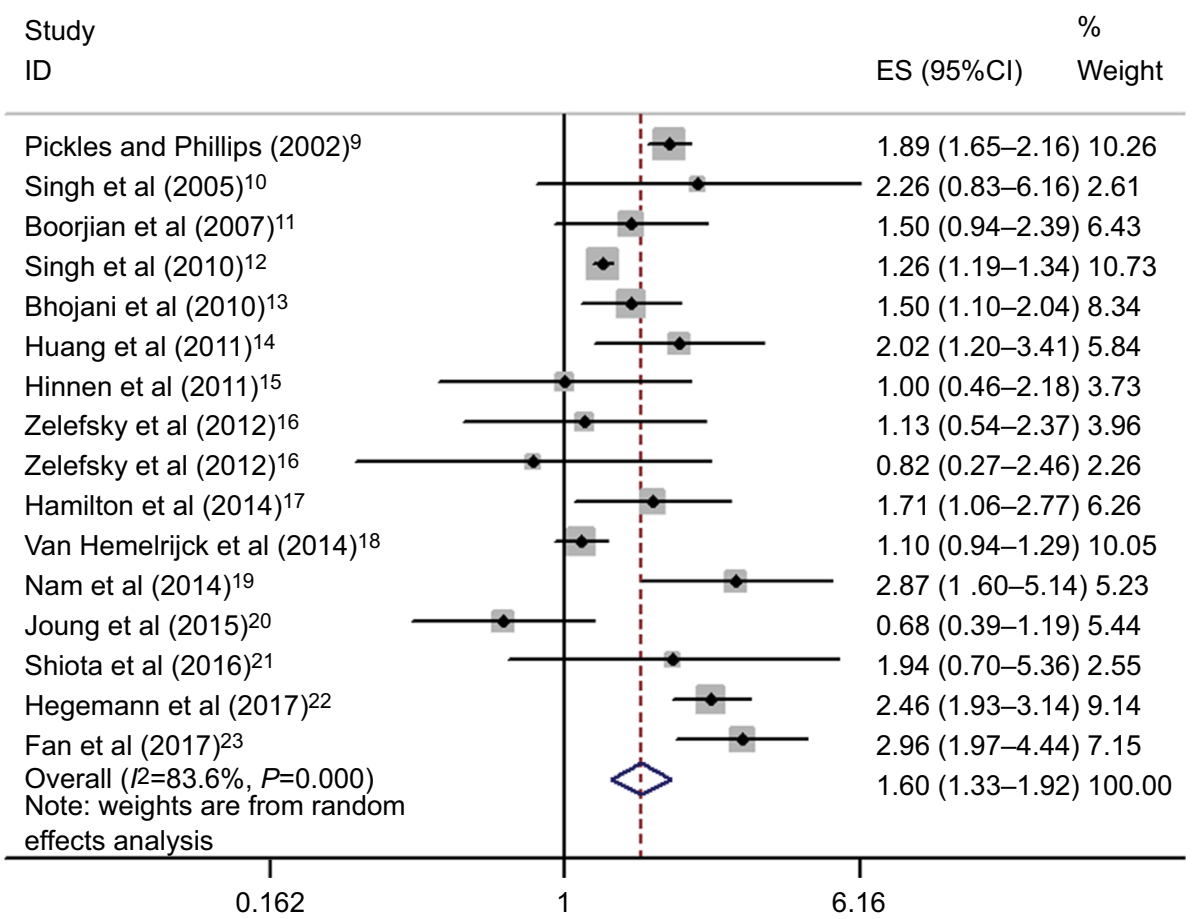

Figure 2 Forest plots of meta-analysis of the included studies on the association between radiotherapy for prostate cancer and secondary bladder cancer. Abbreviation: ES, effect size.

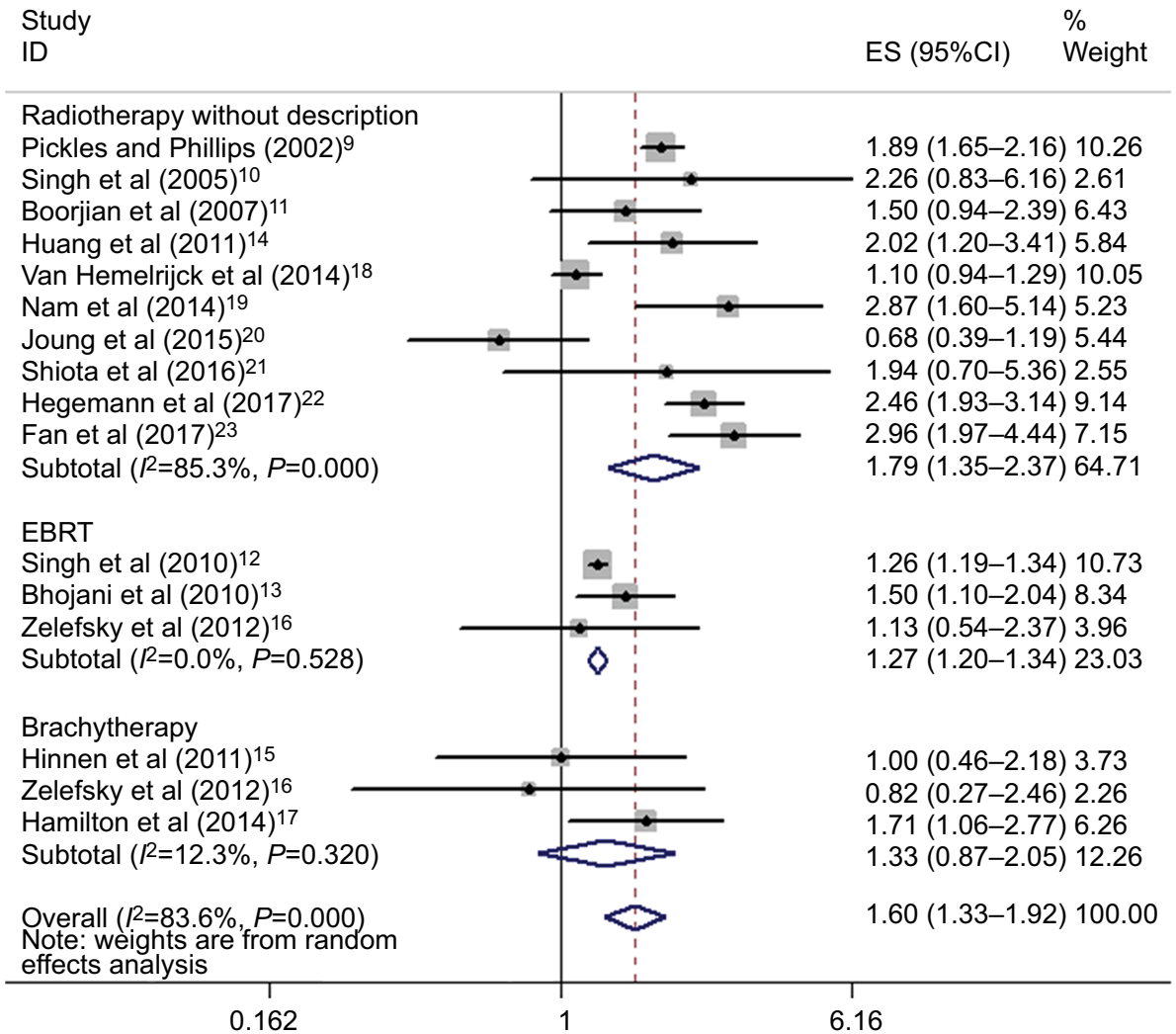

Figure 3 Stratified analysis on the type of radiotherapy.

Abbreviations: EBRT, external beam radiotherapy; ES, effect size. 


\section{Subgroup analyses}

To further elicit the association between RT for PCa and the risk of BLCa and explore the great heterogeneity, subgroup analyses were performed subsequently based on the type of RT, age, geographical area, number of patients, and median follow-up (Table 3). Stratified analysis by the type of RT revealed that both RT without further specification (without reported outcomes of the specific form of RT in some included studies) and EBRT exhibited increased odds of BLCa compared with no RT $(\mathrm{HR}=1.79,95 \% \mathrm{CI}$ : 1.35-2.37; $\mathrm{HR}=1.27$, 95\%CI: 1.2-1.34), whereas when limited to BT did not show this association $(\mathrm{HR}=1.33,95 \% \mathrm{CI}$ : 0.87-2.05). Furthermore, no substantial heterogeneity was detected in EBRT and BT subgroup ( $I^{2}=0 \%$, and $12.3 \%$, respectively) (Figure 3).

Although there is evidence of the RT in causing secondary BLCa overall (pooled HR=1.6, 95\%CI: 1.33-1.92), subgroup analyses showed that compared with RP or non-RT, RT did not significantly increase the occurrence of secondary BLCa for any of the subgroups, i.e., Europe (three studies; ${ }^{15,18,22}$ $\mathrm{HR}=1.45$, 95\%CI: 0.76-2.76, $P=0.261$ ), Asia (three studies: $\left.{ }^{20,21,23} \mathrm{HR}=1.57,95 \% \mathrm{CI}: 0.56-4.44, P=0.394\right)$, and median follow-up $<5$ years (four studies, ${ }^{11,20,21,23} \mathrm{HR}=1.55,95 \% \mathrm{CI}$ : 0.79-3.06, $P=0.202$ ) (Table 3).

\section{Sensitivity analysis}

Sensitivity analysis was performed to evaluate the influence of individual study on the overall risk of BLCa. There was no substantial change on the overall combined HRs, which ranged from 1.52 (95\%CI: $1.27-1.83)$ to $1.68(95 \% \mathrm{CI}$ : 1.39-2.02) after excluding any of the studies (Table 4 and Figure S4). In addition, similar heterogeneity was found after each exclusion, the $I^{2}$ ranged from $78.7 \%$ to $84.7 \%$. The results demonstrated that no single study dominated the combined HRs and heterogeneity.

\section{Publication bias}

Nonsignificant publication bias among the studies was found and confirmed using the Begg's rank correlation test

Table 4 Sensitivity analysis after each study was excluded by turns

\begin{tabular}{|c|c|c|c|}
\hline \multirow[t]{2}{*}{ Study omitted } & \multirow{2}{*}{$\begin{array}{l}\mathrm{HR}(95 \% \mathrm{Cl}) \text { for } \\
\text { remainders }\end{array}$} & \multicolumn{2}{|c|}{ Heterogeneity } \\
\hline & & $I^{2}(\%)$ & $P$ \\
\hline Pickles and Phillips $(2002)^{9}$ & $1.57(1.29-1.91)$ & 79.3 & $<0.001$ \\
\hline Singh et al $(2005)^{10}$ & $1.58(1.31-1.91)$ & 84.5 & $<0.001$ \\
\hline Boorjian et al (2007)" & $1.60(1.32-1.95)$ & 84.7 & $<0.001$ \\
\hline Singh et al $(2010)^{12}$ & $1.63(1.31-2.03)$ & 78.7 & $<0.001$ \\
\hline Bhojani et al $(20 \mid 0)^{13}$ & $1.60(1.31-1.96)$ & 84.6 & $<0.001$ \\
\hline Huang et al $(20 \mathrm{II})^{14}$ & $1.57(1.30-1.90)$ & 84.3 & $<0.001$ \\
\hline Hinnen et al $(20 \mathrm{II})^{15}$ & $1.62(1.34-1.96)$ & 84.6 & $<0.001$ \\
\hline Zelefsky et al $(2012)^{16}$ & $1.62(1.34-1.96)$ & 84.6 & $<0.001$ \\
\hline Zelefsky et al $(2012)^{16}$ & $1.62(1.34-1.96)$ & 84.5 & $<0.001$ \\
\hline Hamilton et al $(2014)^{17}$ & $1.59(1.31-1.93)$ & 84.6 & $<0.001$ \\
\hline Van et al $(20 \mid 4)^{18}$ & $1.66(1.35-2.04)$ & 83.1 & $<0.001$ \\
\hline Nam et al $(2014)^{19}$ & $1.55(1.28-1.86)$ & 83.6 & $<0.001$ \\
\hline Joung et al $(2015)^{20}$ & $1.68(1.39-2.02)$ & 83.6 & $<0.001$ \\
\hline Shiota et al $(2016)^{21}$ & $1.59(1.31-1.92)$ & 84.6 & $<0.001$ \\
\hline Hegemann et al $(2017)^{22}$ & $1.53(1.27-1.83)$ & 79.7 & $<0.001$ \\
\hline Fan et al $(2017)^{23}$ & $1.52(1.27-1.83)$ & 82.0 & $<0.001$ \\
\hline
\end{tabular}

Table 3 Subgroup analysis of the association between RT and secondary BLCa

\begin{tabular}{|c|c|c|c|c|c|}
\hline \multirow[t]{2}{*}{ Category of variables } & \multirow{2}{*}{$\begin{array}{l}\text { No. of } \\
\text { studies }\end{array}$} & \multicolumn{2}{|c|}{ Heterogeneity } & \multicolumn{2}{|c|}{ Random-effects model } \\
\hline & & $I^{2}(\%)$ & $P$ & HR (95\%Cl) & $P$ \\
\hline Radiotherapy without specification & 10 & 85.3 & 0.000 & $1.79(1.35-2.37)$ & $<0.001$ \\
\hline EBRT & 3 & 0.0 & 0.528 & I.27(I.20-I.34) & $<0.001$ \\
\hline Brachytherapy & 3 & 12.3 & 0.32 & $1.33(0.87-2.05)$ & 0.188 \\
\hline \multicolumn{6}{|l|}{ Median follow-up } \\
\hline$<5$ years & 4 & 83.1 & 0.000 & $1.55(0.79-3.06)$ & 0.202 \\
\hline$\geq 5$ years & 9 & 85.8 & 0.000 & $1.59(1.26-2.02)$ & $<0.001$ \\
\hline \multicolumn{6}{|l|}{ Age } \\
\hline$<70$ years & 10 & 79.5 & 0.000 & $1.48(1.13-1.94)$ & 0.005 \\
\hline$\geq 70$ years & 6 & 86.4 & 0.000 & $1.77(1.27-2.46)$ & 0.001 \\
\hline \multicolumn{6}{|l|}{ Geographical area } \\
\hline America & 10 & 78.2 & 0.000 & $1.62(1.31-2.00)$ & $<0.001$ \\
\hline Europe & 3 & 93.3 & 0.000 & I.45 (0.76-2.76) & 0.261 \\
\hline Asia & 3 & 88.5 & 0.000 & $1.57(0.56-4.44)$ & 0.394 \\
\hline \multicolumn{6}{|l|}{ No. of patients } \\
\hline$<10,000$ & 9 & 26.1 & 0.212 & $1.36(1.10-1.67)$ & 0.004 \\
\hline$\geq 10,000$ & 7 & 92.2 & 0.000 & $1.76(1.32-2.34)$ & $<0.001$ \\
\hline
\end{tabular}

Abbreviations: EBRT, external beam radiotherapy; BLCa, bladder cancer; RT, radiotherapy. 
and Egger's linear regression (Begg's, $P>|\mathrm{z}|=0.753$; Egger, $P>|\mathrm{t}|=0.172,95 \% \mathrm{CI}:-0.57-2.88)$ (Figure S5)

\section{Discussion}

It was suggested that the mutagenic potential of ionizing radiation of RT results in the development of second malignancy, especially in the adjacent organ of the prostate, such as the bladder. Over the past few decades, a growing number of cohort studies have examined the association between RT for $\mathrm{PCa}$ and risk of secondary BLCa but provided inconsistent results. Based on data from 15 relevant cohort studies, the current meta-analysis indicates that RT for PCa is associated with secondary BLCa compared to RP treatment or no radiotherapy. PCa patients who received RT might have more than a $60 \%$ increase in the prevalence of secondary BLCa when compared to patients with non-RT. Ascertained by GRADEpro, the rate of events of BLCa on average in patients with RT for prostate cancer and non-RT subjects was $1.3 \%$ and $1.1 \%$, respectively. These results were in line with some pertinent studies, which reported the suspicious association between RT for prostate cancer and BLCa but failed to meet our predefined eligibility criteria. ${ }^{3,8,24-30}$ Moreover, some researchers also found that there was a dose-response relationship between RT and BLCa. ${ }^{31,32}$ In our study, sensitivity analyses revealed that the quantification of the risk for the BLCa in patients with RT for prostate cancer remained prominently higher in nearly all of the included studies, indicating that our findings were robust. We acknowledged that substantial heterogeneity has also been presented in the current meta-analysis. The lag time duration, the different types of RT, the chosen dose of RT, geographical area, median follow-up, adjustment factors, comorbidity, and varied characteristics of subjects could all be partly responsible for the obvious heterogeneity.

The results of the present study were similar with a metaanalysis reported by Wallis et al, ${ }^{30}$ who found that there was a significantly increased risk of BLCa (adjusted $\mathrm{HR}=1.67$, 95\%CI: 1.55-1.80) after RT compared with the risk in those unexposed to RT. Similarly, they also found this relationship when restricted to a 5-year lag period. Moreover, this positive association existed in the analysis of the 10-year lag period in their study, which was quite different from what we found in our study. This might contribute to the number of the included studies (Wallis' study: two studies; current study: four studies), and thus resulted in different overall HR. Another explanation for this inconsistency could be the long interval of time after treatment by RT or RP. It is known that most cancers were age-related factors. This is also likely valuable as the incidence of BLCa increases with aging. Thus, the prevalence of BLCa after a 10-year lag period between RT and RP corresponded closely to each other might probably be due to advanced age.

Although the current meta-analysis has linked RT for prostate cancer to secondary BLCa, it is noteworthy that a certain point in time for the BLCa detection was different among the included studies. It was suggested that the time (lag period) should elapse between the date of received RT intervention (the time of exposure to radiation) and the development of a secondary cancer, and that the tumor might be considered induced by the radiation. ${ }^{33}$ According to the previous studies, the lag period has been defined as 5 years. ${ }^{33,34}$ In the current study, the combined HR from the four included studies provided the 5-year lag period and demonstrated that patients who underwent RT for prostate cancer had an elevated prevalence of secondary BLCa than those who received RP therapy or non-RT intervention. These findings suggested that the secondary BLCa might be induced by radiation. Interestingly, however, it was inconsistent with the above outcomes when restricted to a 10 -year lag period. The four studies reporting the 10-year lag period showed no association between $\mathrm{RT}$ and secondary $\mathrm{BLCa}(\mathrm{HR}=1.93,95 \% \mathrm{CI}$ : 0.9-4.16, $P=0.093)$. Notably, two of four studies ascertained the relationship between RT and secondary BLCa, while the other two studies failed to find a positive association, thus a significant heterogeneity $\left(I^{2}=93.8 \%, P<0.001\right)$ was found as expected. As a result, such evidence was low and further studies are still needed to validate this association. We also proceeded with subgroup analyses to investigate the degree to which potential confounders may influence our findings.

In the subgroup analysis on the type of RT, with the exception of RT without further specification of some included studies, a positive association between RT and BLCa was detected in the EBRT groups whereas no association was found in the BT groups. These results were consistent with a recent study developed by Cosset et al, ${ }^{35}$ they failed to detect any excess risk of secondary BLCa associated with permanent BT with a median follow-up over 11 years. Within the EBRT category, intensity-modulated radiation treatment (IMRT), volumetric modulated arc therapy, 2D- and 3D-conformal X-ray therapy (2D/3D-CRT) are developed conformal RT modalities designed for reducing the local radiationinduced toxicities, but secondary radiation-induced bladder tumors were still occasionally reported by studies. ${ }^{16,19,36,37}$ It was reported that the risk of second malignancy differed remarkably between IMRT and conventional RT for PCa, as well as between different IMRT methods. ${ }^{38}$ They found that 
the conservative maximum risk of secondary malignancy was $1.7 \%, 2.1 \%$ and $5.1 \%$ in conventional radiation, $10-\mathrm{MV}$ $\mathrm{X}$-rays, and 18-MV X-rays of IMRT, respectively. Proton therapy, an emerging treatment modality for EBRT, have consistently shown reduced secondary cancer risks compared with IMRT and 3D-CRT due to the reduction in the volume of normal tissues irradiated. ${ }^{39}$

However, some have even reported that the risk of developing a secondary cancer was lower with either low dose or high dose rate BT alone when compared to any EBRT technique. ${ }^{30}$ When further focused on the dose of $\mathrm{BT}$, Murray et $\mathrm{al}^{40}$ revealed that $\mathrm{PCa}$ patients who received a low dose rate of BT resulted in lower BLCa risks than high dose rate. Taken together, it is likely to recommend younger and healthier patients who did not accept RP treatment for reasons to select BT.

Stratified analysis by median follow-up showed that follow-up over 5 years exhibited increased odds of BLCa after RT, while those limited to $<5$ years did not show this association. As mentioned previously, secondary malignancies were defined as a minimum lag time of 5 years. Thus, researchers might not find such association within 5-year follow-up. Likewise, in a study with a 48-month median follow-up, Okajima et $\mathrm{al}^{41}$ proved the observed incidence of BLCa after RT was higher than the estimated incidence, but not significantly.

It is reported that the incidence of BLCa, especially the muscle-invasive BLCa, increases steadily with age. ${ }^{42}$ Consistent with subgroup analysis on age in the present study, we found that patients aged over 70 years had a higher risk of BLCa compared with those under 70 years old (HR: 1.77 vs 1.48 ). In stratified analysis of the geographical area, an association between BLCa and RT was observed in the US, whereas this association was not found in Europe and Asia. On account of only three studies being included in both Europe and Asia stratification, great existing heterogeneity $\left(I^{2}=93.3 \%, 88.5 \%\right)$ and without providing the adjusted HR, thus this evidence was less robust. When stratified by the total number of patients, higher odds was observed in the subgroup of over 10,000 participants than below 10,000 participants (HR: 1.76 vs 1.36). These outcomes might contribute to the propensity of large sample size within the studies, which affords a higher level of evidence, thus representing the actual incidence of BLCa after RT.

Since evidence has shown an association between RT and secondary primary BLCa, might be potentially lifethreatening. Based on all available data related to the survival analysis of BLCa after RT, the evidence is still controversial.
Of note, it has been reported that in patients with localized $\mathrm{PCa}, 10$-year overall survival was higher in EBRT compared with RP treatment ( $89 \%$ vs $83 \%) .{ }^{43}$ In the cases of treatmentinduced secondary primary BLCa, it was suggested that RT was correlated with poor survival compared to surgical therapy for PCa patients. According to the studies developed by Shiota et al, ${ }^{8,21} 5$-year and 10-year BLCa-free survival of the PCa patients was $97.3 \%$ and $99.4 \%$, and $91.3 \%$ and $97.4 \%$ for EBRT and RP treatment, respectively. These results agreed with the data of Bhojani et al: ${ }^{13}$ patients treated with RT and surgical therapy enabled 10-year BLCa-free survival rate of $96.2 \%$ and $97.3 \%$, respectively. Furthermore, Abern et a ${ }^{28}$ reported that prior RT for $\mathrm{PCa}$ was associated with decreased BLCa-specific survival compared to RP treatment. Interestingly, however, these findings contrast with reports in which patients with PCa who underwent RT were found to have a higher secondary BLCa survival rate compared with RP therapy. Boorjian et $\mathrm{al}^{11}$ reported that patients who received RT and RP therapy presented 5-year BLCa-free survival of $99.1 \%$ and $98.5 \%$, respectively. Singh et al ${ }^{12}$ analyzed over 120,000 men who received RT treatment and 230,000 men with RP therapy for PCa from the SEER database. They found that the number of secondary BLCa deaths was 268/1,836 (14.6\%) and 510/2,753 (22.9\%) in EBRT and RP treatment cohorts, respectively. This is in line with the finding of Hamilton et $\mathrm{al}^{17}$ that deaths from secondary BLCa between BT and RP therapy were 5/33 (15\%) and 9/32 (28\%), respectively.

In this study, we have investigated the potential association between RT and secondary BLCa based on all available evidence published in recent years. We have acknowledged some inherent limitations to our study. First, although all the included studies have defined the secondary malignancies as 5 years after radiation within their studies, only four studies provided the data of 5-year, 10-year lag period. Thus, future studies with over 5-year lag period are required to validate the evidence of RT predisposing to the development of secondary BLCa. Second, it is known that the confounding factors (ie, age, smoking, comorbidities, and other risk factors) could modulate the secondary BLCa risk. However, multivariable adjustments of HRs were only available in four studies. Third, lack of radiotherapy dose and retrospective nature of data used in the analysis with lack of robust long-term follow-up to assess the incidence of BLCa following radiotherapy should also be considered as limitations of the present study. Fourth, we have observed substantial heterogeneity across included studies. Therefore, subgroup analysis and sensitivity analyses were subsequently performed to explore the potential causes of the heterogeneity. And we found that no significant 
heterogeneity was detected when we performed the stratification analyses in EBRT and BT subgroups.

\section{Conclusion}

Our study indicates a higher hazardous effect of RT for developing secondary BLCa compared to RP or non-RT. It is important to note that no such association was observed between BT and secondary BLCa. Though there was an increased risk of BLCa after RT, the absolute rates remained low. And whether the secondary BLCa serves as a lifethreatening complication is still controversial.

\section{Acknowledgments}

This work was supported by the grants from National Natural Science Foundation of China (No. 81372774, No. 81572537 for Zhigang Zhao), The Key Program of Natural Science Foundation of Guangdong Province (No. 2015A030311007 for Zhigang Zhao), Science and Technology Program of Guangzhou (No. 201607010376 for Zhigang Zhao), and The Major Program of Guangdong Education Department (No. 2017KZDXM067 for Zhigang Zhao).

\section{Disclosure}

The authors report no conflicts of interest in this work.

\section{References}

1. Cooperberg MR, Broering JM, Carroll PR. Time trends and local variation in primary treatment of localized prostate cancer. J Clin Oncol. 2010;28(7):1117-1123.

2. Viswanathan AN, Yorke ED, Marks LB, Eifel PJ, Shipley WU. Radiation dose-volume effects of the urinary bladder. Int J Radiat Oncol Biol Phys. 2010;76(3):S116-S122.

3. Bostrom PJ, Soloway MS, Manoharan M, Ayyathurai R, Samavedi S. Bladder cancer after radiotherapy for prostate cancer: detailed analysis of pathological features and outcome after radical cystectomy. J Urol. 2008;179(1):91-95.

4. Abdel-Wahab M, Reis IM, Wu J, Duncan R. Second primary cancer risk of radiation therapy after radical prostatectomy for prostate cancer: an analysis of SEER data. Urology. 2009;74(4):866-871.

5. Narayanan PK, Goodwin EH, Lehnert BE. Alpha particles initiate biological production of superoxide anions and hydrogen peroxide in human cells. Cancer Res. 1997;57(18):3963-3971.

6. Kamran SC, Harshman LC, Bhagwat MS, et al. Characterization of efficacy and toxicity after high-dose pelvic reirradiation with palliative intent for genitourinary second malignant neoplasms or local recurrences after full-dose radiation therapy in the pelvis: a high-volume cancer center experience. Adv Radiat Oncol. 2017;2(2):140-147.

7. Chrouser K, Leibovich B, Bergstralh E, Zincke H, Blute M. Bladder cancer risk following primary and adjuvant external beam radiation for prostate cancer. J Urol. 2005;174(1):107-110.

8. Shiota M, Yokomizo A, Takeuchi A, et al. Secondary bladder cancer after anticancer therapy for prostate cancer: reduced comorbidity after androgen-deprivation therapy. Oncotarget. 2015;6(16):14710-14719.

9. Pickles T, Phillips N. The risk of second malignancy in men with prostate cancer treated with or without radiation in British Columbia, 1984-2000. Radiother Oncol. 2002;65(3):145-151.
10. Singh A, Kinoshita Y, Rovito PM Jr, et al. Higher than expected association of clinical prostate and bladder cancers. J Urol. 2005;173(5): $1526-1529$.

11. Boorjian S, Cowan JE, Konety BR, et al; Cancer of the Prostate Strategic Urologic Research Endeavor Investigators. Bladder cancer incidence and risk factors in men with prostate cancer: results from Cancer of the Prostate Strategic Urologic Research Endeavor. J Urol. 2007;177(3):883-887.

12. Singh AK, Mashtare TL, Mccloskey SA, Seixas-Mikelus SA, Kim HL, May KS. Increasing age and treatment modality are predictors for subsequent diagnosis of bladder cancer following prostate cancer diagnosis. Int J Radiat Oncol Biol Phys. 2010;78(4):1086-1094.

13. Bhojani N, Capitanio U, Suardi N, et al. The rate of secondary malignancies after radical prostatectomy versus external beam radiation therapy for localized prostate cancer: a population-based study on 17,845 patients. Int J Radiat Oncol Biol Phys. 2010;76(2):342-348.

14. Huang J, Kestin LL, Ye H, Wallace M, Martinez AA, Vicini FA. Analysis of second malignancies after modern radiotherapy versus prostatectomy for localized prostate cancer. Radiother Oncol. 2011;98(1): $81-86$.

15. Hinnen KA, Schaapveld M, van Vulpen M, et al. Prostate brachytherapy and second primary cancer risk: a competitive risk analysis. $J$ Clin Oncol. 2011;29(34):4510-4515.

16. Zelefsky MJ, Pei X, Teslova T, et al. Secondary cancers after intensitymodulated radiotherapy, brachytherapy and radical prostatectomy for the treatment of prostate cancer: incidence and cause-specific survival outcomes according to the initial treatment intervention. BJU Int. 2012;110(11):1696-1701.

17. Hamilton SN, Tyldesley S, Hamm J, et al. Incidence of second malignancies in prostate cancer patients treated with low-dose-rate brachytherapy and radical prostatectomy. Int J Radiat Oncol Biol Phys. 2014;90(4):934-941.

18. Van Hemelrijck M, Feller A, Garmo H, et al. Incidence of second malignancies for prostate cancer. PLoS One. 2014;9(7):e102596.

19. Nam RK, Cheung P, Herschorn S, et al. Incidence of complications other than urinary incontinence or erectile dysfunction after radical prostatectomy or radiotherapy for prostate cancer: a population-based cohort study. Lancet Oncol. 2014;15(2):223-231.

20. Joung JY, Lim J, Oh CM, et al. Risk of second primary cancer among prostate cancer patients in Korea: a population-based cohort study. PLoS One. 2015;10(10):e0140693.

21. Shiota M, Yokomizo A, Takeuchi A, et al. Smoking effect on secondary bladder cancer after external beam radiotherapy for prostate cancer. Jpn J Clin Oncol. 2016;46(10):952-957.

22. Hegemann NS, Schlesinger-Raab A, Ganswindt U, et al. Risk of second cancer following radiotherapy for prostate cancer: a population-based analysis. Radiat Oncol. 2017;12(1):2.

23. Fan CY, Huang WY, Lin CS, et al. Risk of second primary malignancies among patients with prostate cancer: a population-based cohort study. Plos One. 2017;12(4):e0175217.

24. Pawlish KS, Schottenfeld D, Severson R, Montie JE. Risk of multiple primary cancers in prostate cancer patients in the Detroit metropolitan area: a retrospective cohort study. The Prostate. 1997;33(2):75-86.

25. Moon K, Stukenborg GJ, Keim J, Theodorescu D. Cancer incidence after localized therapy for prostate cancer. Cancer. 2006;107(5): 991-998.

26. Abdel-Wahab M, Reis IM, Hamilton K. Second primary cancer after radiotherapy for prostate cancer-a SEER analysis of brachytherapy versus external beam radiotherapy. Int J Radiat Oncol Biol Phys. 2008;72(1):58-68.

27. Nieder AM, Porter MP, Soloway MS. Radiation therapy for prostate cancer increases subsequent risk of bladder and rectal cancer: a population based cohort study. J Urol. 2008;180(5):2005-2009.

28. Abern MR, Dude AM, Tsivian M, Coogan CL. The characteristics of bladder cancer after radiotherapy for prostate cancer. Urol Oncol. 2013;31(8):1628-1634. 
29. Davis EJ, Beebe-Dimmer JL, Yee CL, Cooney KA. Risk of second primary tumors in men diagnosed with prostate cancer: a populationbased cohort study. Cancer. 2014;120(17):2735-2741.

30. Wallis CJ, Mahar AL, Choo R, et al. Second malignancies after radiotherapy for prostate cancer: systematic review and meta-analysis. $B M J$. 2016;352:i851.

31. Takam R, Bezak E, Yeoh EE. Risk of second primary cancer following prostate cancer radiotherapy: DVH analysis using the competitive risk model. Phys Med Biol. 2009;54(3):611-625.

32. Schneider U, Lomax A, Besserer J, Pemler P, Lombriser N, Kaser-Hotz B. The impact of dose escalation on secondary cancer risk after radiotherapy of prostate cancer. Int J Radiat Oncol Biol Phys. 2007;68(3):892-897.

33. Murray L, Henry A, Hoskin P, Siebert FA, Venselaar J; PROBATE group of GEC ESTRO. Second primary cancers after radiation for prostate cancer: a systematic review of the clinical data and impact of treatment technique. Radiother Oncol. 2014;110(2):213-228.

34. Sale KA, Wallace DI, Girod DA, Tsue TT. Radiation-induced malignancy of the head and neck. Otolaryngol Head Neck Surg. 2004;131(5):643-645.

35. Cosset JM, Belin L, Wakil G, et al. Second malignancies after permanent implant prostate cancer brachytherapy: a single-institution study of 675 patients treated between 1999 and 2003. Cancer Radiother. 2017;21(3):210-215.

36. Wang B, Xu XG. Measurements of non-target organ doses using MOSFET dosemeters for selected IMRT and 3D CRT radiation treatment procedures. Radiat Prot Dosimetry. 2008;128(3):336-342.
37. Murray LJ, Thompson CM, Lilley J, et al. Radiation-induced second primary cancer risks from modern external beam radiotherapy for early prostate cancer: impact of stereotactic ablative radiotherapy (SABR), volumetric modulated arc therapy (VMAT) and flattening filter free (FFF) radiotherapy. Phys Med Biol. 2015;60(3):1237-1257.

38. Kry SF, Salehpour M, Followill DS, et al. The calculated risk of fatal secondary malignancies from intensity-modulated radiation therapy. Int J Radiat Oncol Biol Phys. 2005;62(4):1195-1203.

39. Cakir A, Akgun Z, Fayda M, Agaoglu F. Comparison of three dimensional conformal radiation therapy, intensity modulated radiation therapy and volumetric modulated arc therapy for low radiation exposure of normal tissue in patients with prostate cancer. Asian Pac J Cancer Prev. 2015;16(8):3365-3370.

40. Murray L, Mason J, Henry AM, et al; UroGEC/BRAPHYQS group of the GEC ESTRO. Modelling second malignancy risks from low dose rate and high dose rate brachytherapy as monotherapy for localised prostate cancer. Radiother Oncol. 2016;120(2):293-299.

41. Okajima K, Ishikawa K, Matsuura T, et al. Multiple primary malignancies in patients with prostate cancer: increased risk of secondary malignancies after radiotherapy. J Clin Oncol Res. 2013;18(6):1078-1084.

42. Fonteyne V, Ost P, Bellmunt J, et al. Curative treatment for muscle invasive bladder cancer in elderly patients: a systematic review. Eur Urol. 2018;73(1):40-50.

43. Kibel AS, Ciezki JP, Klein EA, et al. Survival among men with clinically localized prostate cancer treated with radical prostatectomy or radiation therapy in the prostate specific antigen era. J Urol. 2012;187(4):1259-1265.
Cancer Management and Research

\section{Publish your work in this journal}

Cancer Management and Research is an international, peer-reviewed open access journal focusing on cancer research and the optimal use of preventative and integrated treatment interventions to achieve improved outcomes, enhanced survival and quality of life for the cancer patient. The manuscript management system is completely online and includes

\section{Dovepress}

a very quick and fair peer-review system, which is all easy to use. Visit http://www.dovepress.com/testimonials.php to read real quotes from published authors. 